\title{
Rate allocation based on spectrum pricing function in collaborative transmission over heterogeneous wireless access networks
}

\author{
Jiaojiao Liu*, Yige Wang and Gang Wei
}

\begin{abstract}
This article studied rate allocation in collaborative transmission over heterogeneous wireless access networks. With the introduced pricing function, which uses the occupied spectrum to describe the cost in data transmission, rate allocation is formulated as a concave optimization problem and the explicit solution has been obtained. Instead of transmitting through all available networks in pursuit of fairness, the proposed rate allocation scheme distributes traffic to available networks in an unbalanced way according to both spectral efficiency and network status. Simulation results have shown that compared with other methods, the new scheme can maximize the total utility gained in collaborative transmission and avoid congestion effectively.
\end{abstract}

\section{Introduction}

Nowadays a mobile terminal (MT) with multiple radio transceivers can connect to different wireless access networks. Meanwhile, applications such as voice, video as well as bulk data may need to be run on the MT simultaneously and some of them are bandwidth-hungry. However, any single type of existing wireless and mobile networks cannot meet these requirements. Therefore, heterogeneous wireless access networks have become more and more attractive and they have been built up as a shortdistance wireless access network to satisfy different kinds of applications, such as wireless meeting rooms, wireless E-education, smart houses, and so on.

Since the traffic flow can be distributed among different available access networks to fulfil one transmission task, the rate allocation problem can be regarded as how to divide the traffic among networks that might be loaded differently. Rate allocation over heterogeneous networks (HetNets) can make full use of available resources in different networks and has been studied in [1-5]. In [1,2], cooperative game theory has been applied to rate control and rate allocation at the network level, where the quality of service (QoS) can be guaranteed as long as the required

\footnotetext{
${ }^{*}$ Correspondence: jjliu@scut.edu.cn

School of Electronic and Information Engineering, South China University of

Technology, 381 Wushan Road, Guangzhou 510640, Guangdong Province,
} China transmission rate is satisfied. The non-cooperative game framework of bandwidth allocation in $4 \mathrm{G}$ heterogeneous wireless access networks has been discussed in [3]. In [4], an $H^{\infty}$-optimal control formulation for allocating rates to devices over multiple access networks with heterogeneous time-varying characteristics has been proposed for the worst-case scenario. To support multiple video streams, a pricing-based mechanism has been introduced to the bitrate allocation [5], where the price is adjusted based on the difference between supply and demand for the current slot. In all the above works, the throughput gain has been chosen as the optimization objective without considering the heterogeneity of spectral efficiency and network status and all the available networks must participate in collaborative transmission. Then the heavy-load network will be easily saturated and packets will be dropped. Furthermore, it makes no sense for a low traffic flow to be distributed to different networks in pursuit of fairness, where the cost of packet disassembling and resembling cannot be ignored.

Therefore, network status should be considered in rate allocation over HetNets. It can be described using the resource utilization since a network is more saturated with more resources occupied. Then similar as [6], a pricing function can be used to allocate the transmission rate according to network status and distribute more traffic to light-load networks to prevent network congestion. In

\section{Springer}

C 2012 Liu et al: licensee Springer. This is an Open Access article distributed under the terms of the Creative Commons

Attribution License (http://creativecommons.org/licenses/by/2.0), which permits unrestricted use, distribution, and reproduction in any medium, provided the original work is properly cited. 
this article we have focused on networks such as WiMax, UWB, Cellular networks, etc., where frequency sub-bands are the resources to be allocated [7]. With the utilized resource described by the occupied spectrum, the pricing function is modelled as the resource cost in data transmission and the utility of an MT can be formulated as the throughput gain minus the resource cost. Then rate allocation becomes a global optimization problem, where the traffic is transmitted at a minimum resource cost, and an explicit solution can be obtained based on the convex optimization theory. It is straightforward to extend our scheme to other cases, such as time-slotted systems, where the pricing function is introduced based on occupied time-slots and the rate allocation problem can be formulated using a similar framework to prevent network congestion.

\section{Model and problem formulation Wireless transmission}

In wireless communications, transmission rate can be adjusted dynamically based on the channel quality using the adaptive modulation. Assume $n$ networks are built up with overlapped coverage. Then the bit error rate (BER) over a single-input single-output Gaussian noise channel in network $i$ for the uncoded quadrature amplitude modulation (QAM) with square constellation can be calculated as follows $[6,8]$

$$
\mathrm{BER}_{i}=0.2 \exp \left(\frac{-1.5 \gamma_{i}}{\left(2^{\varepsilon_{i}}-1\right)}\right)
$$

where $\gamma_{i}$ is the signal to noise ratio (SNR) at the receiver and $\varepsilon_{i}$ denotes the spectral efficiency of the selected modulation scheme in network $i$. To guarantee the quality of transmission, BER should not exceed a certain level, denoted by BER $R_{i}^{\text {tar }}$. Then the spectral efficiency of transmission for an MT in network $i$ can be obtained from

$$
\varepsilon_{i}=\log _{2}\left(1+\frac{1.5}{\ln \left(0.2 / \mathrm{BER}_{i}^{\mathrm{tar}}\right)} \gamma_{i}\right)
$$

Assume that the value of BER is available at the transmitter by using channel estimation. Then the transmission rate $r_{i}$ (in bits per second) can be calculated according to the occupied spectrum $f_{i}$, i.e.,

$$
r_{i}=\varepsilon_{i} f_{i} .
$$

\section{Model and notation}

Assume that $N$ HetNets are available to an MT. Let the available bit rate (ABR) in network $i$ be $\mu_{i}$ for $i=1, \ldots, N$, which can be measured via online measurement tools [4] or estimated using the Markov model [9]. For the $k$ th incoming traffic flow $v_{k}$ in the coverage area, the strategy in network $i$ corresponds to the allocated rate $x_{i, k}$, where $0<x_{i, k}<\mu_{i}$ and the utility gained in transmission is a function of $x_{i, k}$, denoted by $U_{i}\left(x_{i, k}\right)$. Then the target of rate allocation is to maximize the global utility, i.e.,

$$
\max _{0 \leq x_{i, k} \leq \mu_{i}} \sum_{i=1}^{N} U_{i}\left(x_{i, k}\right) \quad \text { s.t. } \quad \sum_{i=1}^{N} x_{i, k}=v_{k} .
$$

For simplicity, the cost of packet disassembling and reassembling has not been considered in this article.

\section{Mathematical formulation}

If there are $M$ traffic flows in network $i$ and $f_{i, j}$ is the occupied spectrum of the $j$ th flow, then similar as [6], the pricing function can be defined as follows

$$
P_{i}(\mathbf{r})=b+\omega\left(\sum_{j=1}^{M} f_{i, j}\right)^{\eta}=b+\omega\left(\sum_{j=1}^{M} c_{i, j} r_{i, j}\right)^{\eta}
$$

where $b$ is the price base, $\omega$ and $\eta$ are used to adjust the growth speed of the price according to the occupied spectrum. Here, $b, \omega$ and $\eta$ are non-negative constants with $\eta \geq 1$ in order to make the pricing function convex. Let $c_{i, j}=\frac{1}{\varepsilon_{i, j}}$, where $\varepsilon_{i, j}$ is the spectral efficiency for the transmission of traffic flow $j$ over network $i$ and $\mathbf{r}_{\mathbf{i}}$ denote a rate vector of the $M$ traffic flows in network $i$, i.e., $\mathbf{r}_{\mathbf{i}}=\left\{r_{i, 1}, \ldots, r_{i, M}\right\}$. As shown in (5), the price is defined as a function of the total occupied spectrum in the coverage area. This means that when a coverage area becomes congested, the spectrum resource will become scarce and higher price will be charged to gain more revenue. Therefore, the nondecreasing pricing function in (5) can avoid saturation in a wireless network [10]. Furthermore, $b, \omega$ and $\eta$ can be set according to specific applications or user preference. For example, the price base $b$ can be the monthly rentals in telecommunication. Then $b=0$ for the free access networks such as WiFi. For networks with fixed spectrum assignment, set $\omega=0$ so that network status is not taken into consideration. The parameter $\eta$ is useful for random access networks, where the price rises fast with the increase of the occupied spectrum. In this article, for simplicity, set $b=0, \omega=1.0$, and $\eta=1.0$ for all networks.

Let $\alpha_{i}$ be the unit throughput gain of network $i$. Without loss of generality, assume among the $M$ traffic flows in network $i$, the $k$ th flow is for the incoming traffic flow, while the others are existing ones. Therefore, $r_{i, k}=x_{i, k}$. Then the revenue for an MT in transmission over network $i$ can be described as the total throughput gain of network $i$, i.e., 
$\alpha_{i} x_{i, k}$ and the utility gained from network $i$ can be defined as the revenue minus the spectrum resource cost, i.e.

$$
U_{i}\left(x_{i, k}\right)=\alpha_{i} x_{i, k}-P_{i}(\mathbf{r}) x_{i, k} .
$$

Then rate allocation over networks can be modelled as a global maximization problem $P_{S}$

$$
\begin{aligned}
& \max _{0 \leq x_{i, k} \leq \mu_{i}} \sum_{i=1}^{N} U_{i}\left(x_{i, k}\right) \\
& \text { s.t. } \sum_{i=1}^{N} x_{i, k}=v_{k} \\
& 0 \leq x_{i, k} \leq \mu_{i}, \quad i=1, \ldots, N
\end{aligned}
$$

Take the first and second partial derivatives of $\sum_{i=1}^{N}$ $U_{i}\left(x_{i, k}\right)$ with respect to $x_{i, k}$, we obtain

$$
\begin{aligned}
\frac{\partial \sum_{i=1}^{N} U_{i}\left(x_{i, k}\right)}{\partial x_{i, k}}= & \alpha_{i}-b-\omega\left(\sum_{j=1}^{M} c_{i, j} r_{i, j}\right)^{\eta} \\
& -\omega \eta c_{i, k}\left(\sum_{j=1}^{M} c_{i, j} r_{i, j}\right)^{\eta-1} \cdot x_{i, k} \\
\frac{\partial^{2} \sum_{i=1}^{N} U_{i}\left(x_{i, k}\right)}{\partial x_{i, k}^{2}}= & -2 \omega \eta c_{i, k}\left(\sum_{j=1}^{M} c_{i, j} r_{i, j}\right)^{\eta-1} \\
& -\omega \eta(\eta-1) c_{i, k}^{2}\left(\sum_{j=1}^{M} c_{i, j} r_{i, j}\right)^{\eta-2} \cdot x_{i, k} .
\end{aligned}
$$

It is easily checked that $\partial^{2} \sum_{i=1}^{N} U_{i}\left(x_{i, k}\right) / \partial x_{i, k}^{2}<0$. Therefore, $\sum_{i=1}^{N} U_{i}\left(x_{i, k}\right)$ is a concave function of $x_{i, k}$ and $P_{S}$ is a convex optimization problem.

Proposition 2.1. Since $\sum_{i=1}^{N} U_{i}\left(x_{i, k}\right)$ is concave in $x_{i, k}$ and has continuous first partial derivative, the optimization problem $P_{S}$ can be formulated as a Lagrangian function, where the first-order Kuhn-Tucker conditions are necessary and sufficient for optimality. Therefore, the solution of (7) can be obtained as follows

$$
\begin{aligned}
x_{i, k}= & \max \left\{0, \min \left[\frac { \varepsilon _ { i , k } } { \sum _ { l = 1 } ^ { N } \varepsilon _ { l , k } } \left(v_{k}+\frac{1}{2} \sum_{l=1}^{N} \varepsilon_{l, k}\left(\alpha_{i}-\alpha_{l}\right)\right.\right.\right. \\
& \left.\left.\left.+\frac{1}{2} \sum_{l=1}^{N} \varepsilon_{l, k} \sum_{j=1, j \neq k}^{M}\left(c_{l, j} r_{l, j}-c_{i, j} r_{i, j}\right)\right), \mu_{i}\right]\right\} .
\end{aligned}
$$

The proof of Proposition 2.1 can be found in the Appendix. In (9), $\sum_{j=1, j \neq k}^{M} c_{l, j} r_{l, j}$ denotes the occupied spectrum in network $l$ before rate allocation and $\varepsilon_{i, k} / \sum_{l=1}^{N} \varepsilon_{l, k}$ is the ratio of the spectral efficiency of the $k$ th traffic transmission in network $i$ to that of all available networks. Therefore, $\sum_{j=1, j \neq k}^{M}\left(c_{l, j} r_{l, j}-c_{i, j} r_{i, j}\right)$ denotes the difference between the occupied spectrum in network $l$ and that in network $i$ before rate allocation, which is used to adjust the allocated rate. However, if $v_{k}+\frac{1}{2} \sum_{l=1}^{N} \varepsilon_{l, k}\left(\alpha_{i}-\alpha_{l}\right)<$ $\frac{1}{2} \sum_{l=1}^{N} \varepsilon_{l, k} \sum_{j=1, j \neq k}^{M}\left(c_{i, j} r_{i, j}-c_{l, j} r_{l, j}\right)$, then $x_{i, k}<0$. That means available spectrum in network $i$ is much less than others and the solution will be feasible by setting $x_{i, k}=0$.

Proposition 2.2. When the networks become identical with the same unit throughput gain and the same occupied spectrum, then

$$
x_{i, k}=\min \left(\frac{\varepsilon_{i, k}}{\sum_{l=1}^{N} \varepsilon_{l, k}} v_{k}, \mu_{i}\right)
$$

Proof. When the unit throughput gain of all networks is the same, $\left(\alpha_{i}-\alpha_{l}\right)=0$. Also $\sum_{j=1, j \neq k}^{M}\left(c_{l, j} r_{l, j}-c_{i, j} r_{i, j}\right)=0$ when the networks are identical with the same occupied spectrum. Then, (9) becomes (10).

Equation (10) the same as the proportional allocation with only the throughput gain as the utility function in the Nash bargaining game[1].

\section{Fairness index of rate allocation}

In order to measure the "equality" of the rate allocated in different networks, a fairness index is defined as that in $[11,12]$.

$$
I\left(\mathbf{R}_{\mathbf{k}}\right)=\frac{\left(\sum_{i=1}^{N} R_{i, k}\right)^{2}}{N \sum_{i=1}^{N} R_{i, k}^{2}},
$$

where $R_{i, k}=\frac{x_{i, k}}{\mu_{i}}$ is the ABR utilization ratio in network $i$ and $\mathbf{R}_{\mathbf{k}}=\left(R_{1, k}, R_{2, k}, \ldots, R_{N, k}\right)$. Obviously, this fairness index is a measure of the balance of the ABR utilization ratio in rate allocation. If all the networks have the same ABR utilization ratio, then $I=1$ and the rates allocated in networks are proportional to their capabilities in a completely balanced way. If $I \neq 1$, some networks will be given preference to transmit.

\section{Performance evaluation Parameter setting}

Assume three networks with overlapped coverage are available to an MT, where frequency sub-bands are the resources to be allocated. To model multimedia traffic in 
Table 1 Parameters in three experiments

\begin{tabular}{llll}
\hline Experiment & $\begin{array}{l}\text { Number of existing } \\
\text { streams in each network }\end{array}$ & $\begin{array}{l}\text { SNR of exsiting streams } \\
\text { in each network }\end{array}$ & $\begin{array}{l}\text { SNR of the incoming } \\
\text { traffic in each network }\end{array}$ \\
\hline 1 & $5,5,5$ & $0.1,0.1,0.1$ & $0.2,0.1,0.07$ \\
2 & $5,5,5$ & $0.2,0.1,0.07$ & $0.2,0.1,0.07$ \\
3 & $5,7,10$ & $0.1,0.1,0.1$ & $0.2,0.2,0.2$ \\
\hline
\end{tabular}

networks, assume video streams with a rate of $64 \mathrm{Kbps}$ and a packet size of 1,000 bytes need to be transmitted through the networks. Let the target BER be the same for all users in any network and set $\mathrm{BER}_{i}^{\mathrm{tar}}=10^{-4}$, for $i=1,2,3$.

\section{Numerical results}

With different access technologies, HetNets are different in network coverage, network status, spectral efficiency and the resulting network capability. Network status can be characterized by the occupied spectrum since a network is more saturated with more spectrum resources utilized. Moreover, the SNR at the receiver is decided by the position of an MT within a network and is related with the spectral efficiency as in (2). Then according to (3), the transmission capability is decided by both SNR and the occupied spectrum. To verify that our scheme allocates rates based on spectral efficiency and network status, we conduct three experiments, where SNR and occupied spectrum are used to describe the heterogeneity of different networks. The parameters of these three experiments are shown in Table 1.
Experiment 1. Three networks have the same occupied spectrum, but different spectral efficiency.

Assume there are five existing streams in each network and they are from $5 \mathrm{MTs}$, respectively. Set the received SNRs of all existing streams to 0.1 , then the occupied spectrum in each network is the same. Suppose the SNRs for a new MT in three networks are 0.2, 0.1, and 0.07, respectively. Then the spectral efficiency for the new MT in each network is different with the largest value in network 1 . Rate allocation is carried out according to (10) and the results are shown in Figure 1. We observe from the figure that when the transmission rate in network 1 is below $300 \mathrm{Kbps}$, the traffic will be distributed to all networks in proportional to their spectral efficiencies respectively and the rate allocated to network 1 is the largest.

Experiment 2. Three networks have different occupied spectrum and different spectral efficiency.

Again assume five existing streams in each netowrk with the received SNRs equal to $0.2,0.1$, and 0.07 , respectively. Then according to (3) the occupied spectrum of these three networks is $5.73,11.35$, and $16.17 \mathrm{KHz}$, respectively. Moreover, the spectral efficiencies for a new MT

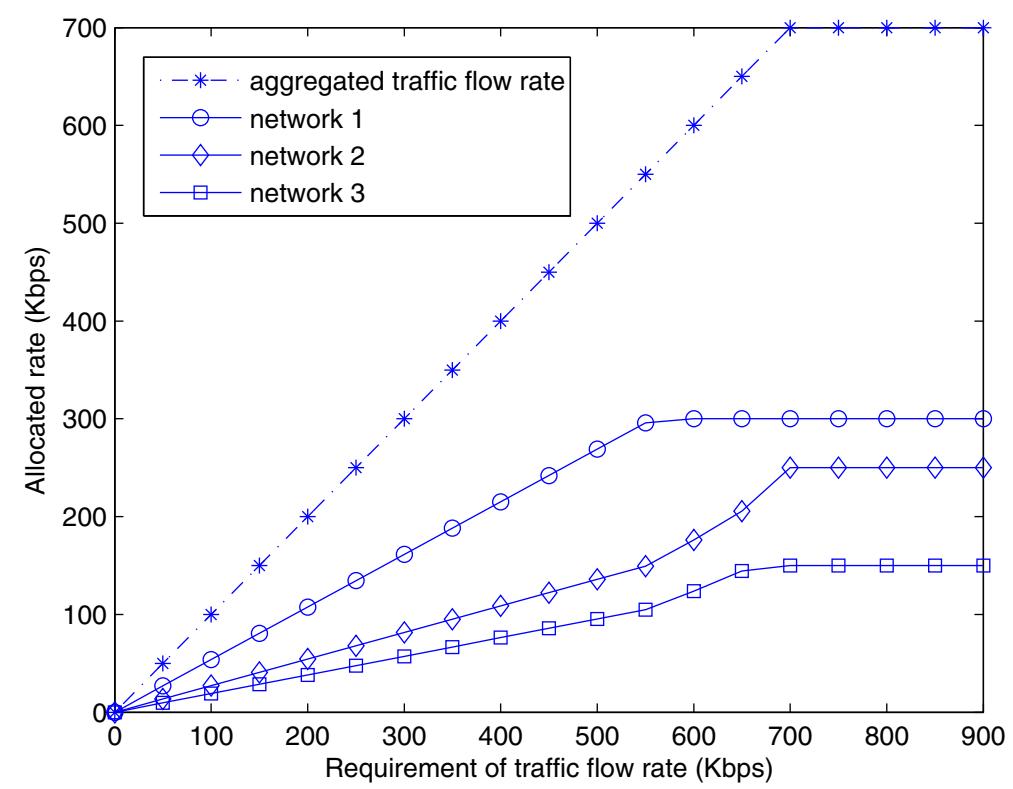

Figure 1 Rate allocation in networks with the same occupied spectrum. 


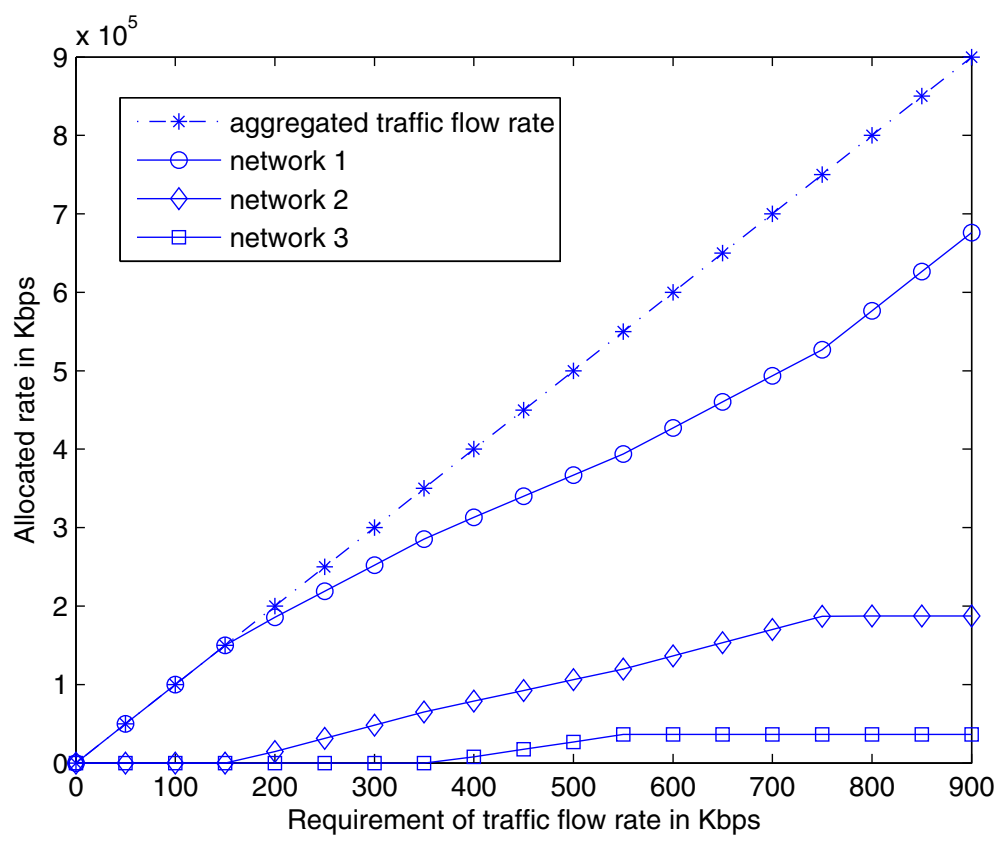

Figure 2 Rate allocation in networks with the same occupied spectrum.

in these networks are assumed to be different. The rate allocation results are shown in Figure 2. We observe that the incoming traffic flow prefers to choose networks with less occupied spectrum and higher spectral efficiency for transmission. Only network 1 is chosen to transmit when the required flow rate is below $150 \mathrm{Kbps}$. When the required flow rate is no more than $350 \mathrm{Kbps}$, network 3 with limited capacity will not transmit to avoid congestion.
Experiment 3. Three networks have different traffic load and the same ABR.

Assume the ABR is the same in different networks with the value of $300 \mathrm{Kbps}$. Let the number of existing streams in different networks be 5,7 , and 10 , respectively, and the SNR be the same for these streams. Then the network load and the occupied spectrum are different for each network. The rate allocation results are shown in Figure 3. We observe that the traffic tends to be distributed to networks

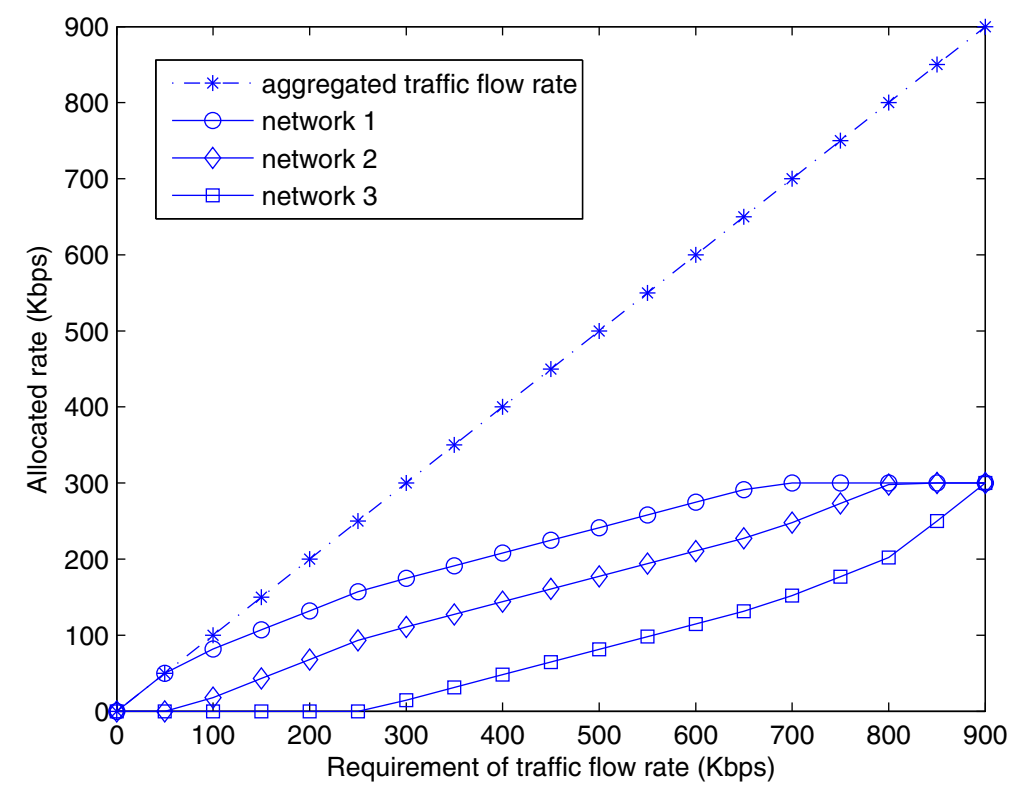

Figure 3 Rate allocation in networks with the same $A B R s(5,7,10$ video streams in network $1,2,3)$. 


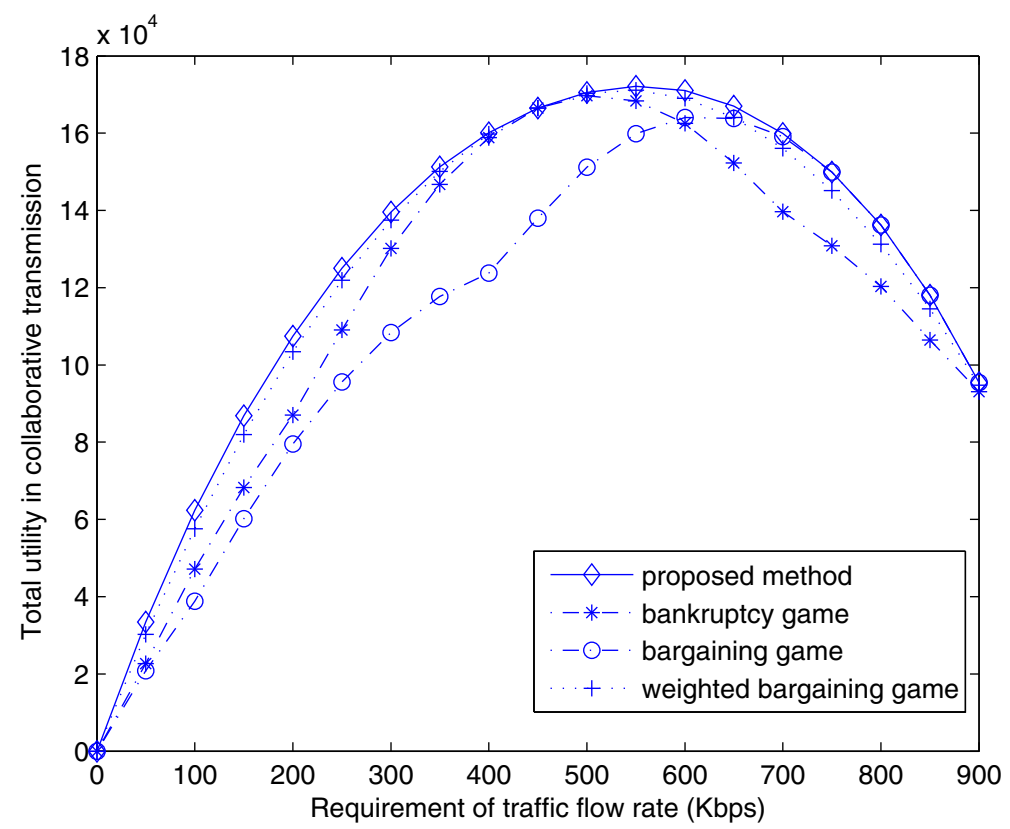

Figure 4 Total utility in collaborative transmission with different methods.

with less traffic load and less occupied spectrum. Network 3 will not transmit until the required flow rate exceeds 250 Kbps.

In summary, the above experiment results show that the proposed method can select one or several networks to transmit data and distribute the traffic to them according to their spectral efficiencies and network status. Our scheme is different from the method in the bargaining game [1] or that based on the bankruptcy game [2]. In the bargaining game, HetNets are rational players and they complete the high-speed transmission in a cooperative way. The players' interests may conflict and an outcome is collectively negotiated to benefit all the individuals. With the global throughput gain as the objective, the bargaining game framework will result in an average allocation. For example, assume the rate of the incoming traffic is

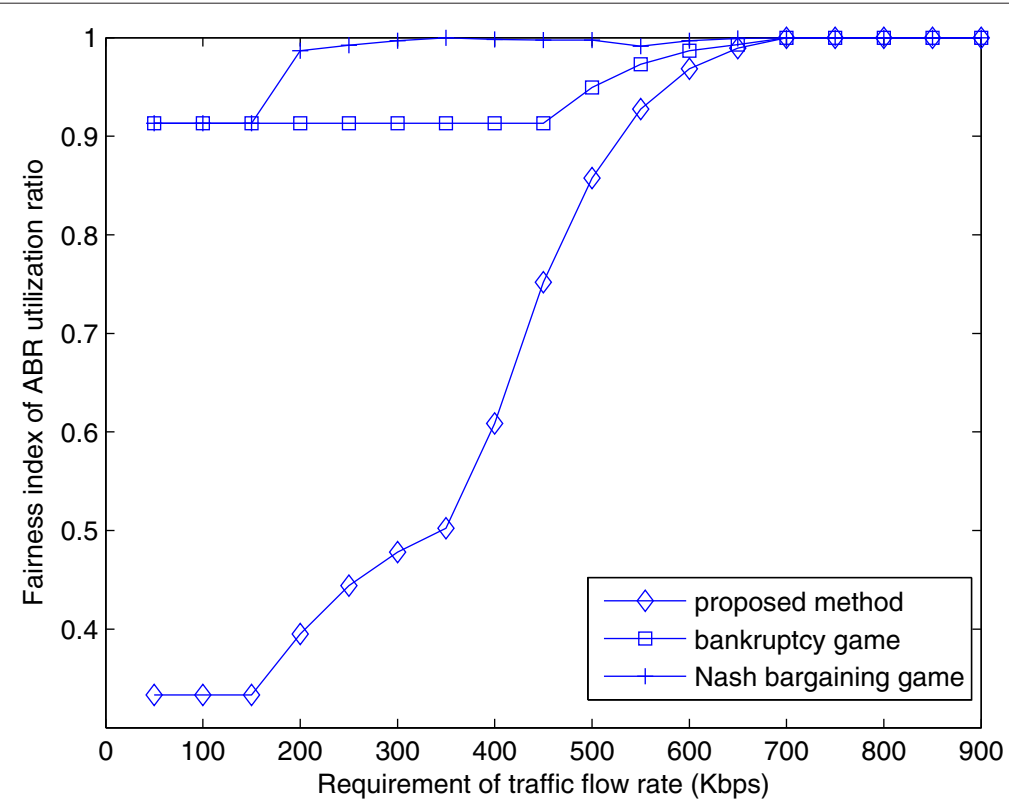

Figure 5 Fairness index of ABR utilization. 
$450 \mathrm{Kbps}$ and the ABRs of three available networks are 300,250 , and $150 \mathrm{Kbps}$, respectively. Then the allocated rate is $150 \mathrm{Kbps}$ for each network, which will exhaust the ABR of network 3 and lead to high packet loss. In the meantime, the ABR utilization ratio of network 1 is only $50 \%$. Obviously, the above problem can be solved if more traffic is distributed to network 1 . In the bankruptcy game, the traffic could be distributed to networks in a similar way as the assignment of asset for creditors in bankruptcy. According to the superadditivity property of the grand coalition, the global payoff will be maximized with the participation of all networks. However, the traffic distributed to heavy-load networks will cause congestion. It is obvious that the network status has not been fully studied in all the above cases, while our scheme can provide a better solution, where a heavy-load network can exit cooperation.

In the real life situation, the occupied spectrum and the spectral efficiency are different for different networks. So the scenario in experiment 2 is more representative. We compare the total utility gained for an MT in the scenario of experiment 2 using our scheme with those obtained by other methods and the results are depicted in Figure 4. As shown in (6) and (7), our scheme is to maximize the throughput gain with the least spectrum cost, so it distributes traffic to light-load networks because of higher price barriers set in heavy-load networks. In the game frameworks above, rate allocation only takes fairness into consideration and the traffic allocated to heavy-load networks will lead to more resource cost. Therefore, these schemes have worse performance compared with ours.

In addition, the fairness index of ABR utilization ratio of different schemes is compared in Figure 5. It has shown that the balance degree of the ABR utilization ratio is the best in the bankruptcy game framework, while it is the worst in the proposed method. This phenomenon implies that the proposed rate allocation scheme improves performance at the cost of fairness.

\section{Conclusions}

Using the occupied spectrum to represent the network status, a nondecreasing pricing function is introduced to describe the resource cost in transmission. To achieve the throughput gain at a minimum resource cost, rate allocation can be formulated as a concave optimization problem and the explicit solution has been obtained. Experiment results have shown that the proposed method can serve as an access selection method, which distributes the traffic according to both spectral efficiency and network status. The results also show that compared with other schemes the proposed method has the highest total utility gained from networks with the traffic distributed in an unbalanced way.

\section{Appendix}

\section{Proof of Proposition 2.1}

As shown in (8), the second partial derivation of $\sum_{i=1}^{N} U_{i}\left(x_{i, k}\right)$ is negative, so it is concave and $\left(P_{S}\right)$ can be solved based on the optimization theory. Moreover, $\sum_{i=1}^{N} U_{i}\left(x_{i, k}\right)$ has a continuous first partial derivation, which implies the first-order Kuhn-Tucker conditions are necessary and sufficient for optimality [13]. Define the Lagrangian function as $L\left(x_{i, k}, \eta, \delta_{i}, \zeta_{i}\right)$, where $\eta \leq 0, \delta_{i} \leq$ $0, \zeta_{i} \leq 0$, for $i=1, \ldots, N$

$$
\begin{aligned}
L\left(x_{i, k}, \eta, \delta_{i}, \zeta_{i}\right)= & \sum_{i=1}^{N}\left[\alpha_{i} x_{i, k}-\left(\sum_{j=1}^{M} c_{i, j} r_{i, j}\right) x_{i, k}\right] \\
& +\eta\left(\sum_{i=1}^{N} x_{i, k}-v_{k}\right) \\
& +\sum_{i=1}^{N} \delta_{i}\left(x_{i, k}-\mu_{i}\right)-\sum_{i=1}^{N} \zeta_{i} x_{i, k}
\end{aligned}
$$

The first-order Kuhn-Tucker conditions are

$\frac{\partial L}{\partial x_{i, k}}=\alpha_{i}-\sum_{j=1, j \neq k}^{M} c_{i, j} r_{i, j}-2 c_{i, k} x_{i, k}+\eta+\delta_{i}-\zeta_{i}, \quad i=1, \ldots, N$

$$
\frac{\partial L}{\partial \eta}=\sum_{i=1}^{N} x_{i, k}-v_{k}
$$

and

$$
\begin{aligned}
& \delta_{i}\left(x_{i, k}-\mu_{i}\right)=0, \delta_{i} \leq 0, \quad i=1, \ldots, N \\
& \zeta_{i} x_{i, k}=0, \zeta_{i} \leq 0, \quad i=1, \ldots, N
\end{aligned}
$$

As the problem $P_{s}$ shows, the value of $x_{i, k}$ is either zero or a positive number, which is no more than $\mu_{i}$. Therefore, three cases are considered below. 
Case 1. $0<x_{i, k}<\mu_{i}$ Equation (15) and (16) imply $\delta_{i}=$ $0, \zeta_{i}=0$ separately. Therefore

$$
\begin{aligned}
& \alpha_{i}-\sum_{j=1, j \neq k}^{M} c_{i, j} r_{i, j}-2 c_{i, k} x_{i, k}+\eta=0, \quad i=1, \ldots, N \\
& \sum_{i=1}^{N} x_{i, k}=v_{k}
\end{aligned}
$$

Then

$$
\begin{aligned}
x_{i, k}= & \frac{\varepsilon_{i, k}}{\sum_{l=1}^{N} \varepsilon_{l, k}}\left(v_{k}+\frac{1}{2} \sum_{l=1}^{N} \varepsilon_{l, k}\left(\alpha_{i}-\alpha_{l}\right)\right. \\
& \left.+\frac{1}{2} \sum_{l=1}^{N} \varepsilon_{l, k} \sum_{j=1, j \neq k}^{M}\left(c_{l, j} r_{l, j}-c_{i, j} r_{i, j}\right)\right), i=1, \ldots, N
\end{aligned}
$$

Case 2. $x_{i, k}=\mu_{i}$

It follows from (15) that $\delta_{i} \leq 0$ and (16) implies $\zeta_{i}=0$. Therefore

$$
\begin{array}{r}
\alpha_{i}-\sum_{j=1, j \neq k}^{M} c_{i, j} r_{i, j}-2 c_{i, k} x_{i, k}+\eta=-\delta_{i} \geq 0, x_{i, k} \leq \mu_{i} \\
\text { So } x_{i, k} \leq \frac{\varepsilon_{i, k}}{\sum_{l=1}^{N} \varepsilon_{l, k}}\left(v_{k}+\frac{1}{2} \sum_{l=1}^{N} \varepsilon_{l, k}\left(\alpha_{i}-\alpha_{l}\right)+\frac{1}{2} \sum_{l=1}^{N}\right. \\
\left.\varepsilon_{l, k} \sum_{j=1, j \neq k}^{M}\left(c_{l, j} r_{l, j}-c_{i, j} r_{i, j}\right)\right)
\end{array}
$$

Case 3. $x_{i, k}=0$

It follows from (16) that $\zeta_{i} \leq 0$ and (15) implies $\delta_{i}=0$. Therefore

$$
\alpha_{i}-\sum_{j=1, j \neq k}^{M} c_{i, j} r_{i, j}-2 c_{i, k} x_{i, k}+\eta=\zeta_{i} \leq 0, x_{i, k} \geq 0
$$

So $v_{k}+\frac{1}{2} \sum_{l=1}^{N} \varepsilon_{l, k}\left(\alpha_{i}-\alpha_{l}\right)+\frac{1}{2} \sum_{l=1}^{N} \varepsilon_{l, k} \sum_{j=1, j \neq k}^{M}$ $\left(c_{l, j} r_{l, j}-c_{i, j} r_{i, j}\right) \leq 0$ and $x_{i k} \leq 0$

Therefore $x_{i k}=0$.

In summary, the solution of $P_{S}$ can be calculated using (9).

\section{Competing interests}

The authors declare that they have no competing interests.

\section{Acknowledgements}

The authors would like to thank the National Natural Science Foundation of China No. U1035003 and the National Basic Research Program of China (973) under Grant No. 2011CB707003, the Guangdong Province Key Laboratory of short distance wireless detection and Communication, the Natural Science Foundation Team Project of Guangdong Province No. 9351064101000003 and the Fundamental Research Funds for the Central Universities of SCUT under grant number 2012 ZM0020.

\section{References}

1. M Khan, C Truong, T Geithner, F Sivrikaya, Network level cooperation for resource allocation in future wireless networks. in Proceedings of the IFIP Wireless Days Conference '08. (Dubai, 1-5, 24-27 Nov 2008)

2. D Niyato, E Hossain, A cooperative game framework for bandwidth allocation in $4 \mathrm{G}$ heterogeneous wireless networks. in IEEE International Conference on Communications. (Istanbul, 4357-4362, 11-15 June 2006)

3. D Niyato, E Hossainm, A noncooperative game-theoretic framework for radio resource management in $4 \mathrm{G}$ heterogeneous wireless access neworks. IEEE Trans. Mobile Comput. 7, 332-345 (2008)

4. TAlpcan, JP Singh, T Basar, Robust rate control for heterogeneous network access in multihomed environments. IEEE Trans. Mobile Comput. 8, 41-51 (2009)

5. M Tiwari, T Groves, P Cosman, Bit-rate allocation for multiple video streams using a pricing-based mechanism. IEEE Trans. Image Process. 20 3219-3230 (2011)

6. D Niyato, E Hossain, A game-theoretic approach to competitive spectrum sharing in cognitive radio networks. in IEEE Wireless Communications \& Networking Conference. (HongKong, 6-20, 11-15 March 2007)

7. A Rajeswaran, G Kim, R Negi, A scheduling framework for UWB \& cellular networks. Mobile Netw. Appl. 1, 9-20 (2006)

8. AJ Goldsmith, SG Chua, Variable rate variable power MQAM for fading channels. IEEE Trans. Commun. 45, 1218-1230 (1997)

9. JP Singh, T Alpcan, P Agrawal, V Sharma, An optimal flow assignment framework for heterogeneous network access. in IEEE International Symposium on a World of Wireless, Mobile and Multimedia Networks 2007. (Helsinki, 1-12, 18-21 June 2007)

10. J Hou, J Yang, S Papavassiliou, Integration of pricing with call admission control to meet qos requirements in cellular networks. IEEE Trans. Parallel Distrib. Syst. 13, 898-910 (2002)

11. D Grosu, A Chronopoulos, L Ming-Ying, Load balancing in distributed systems: an approach using cooperative games. in Proceedings of the International Parallel and Distributed Processing Symposium 2002. (Lauderdale, 52-61, 15-19 Apr 2002)

12. R Jain, D Chiu, W Hawe, A Quantitative Measure of Fairness and Discrimiation for Resource Allocation in Shared Computer System. (Eastern Research Lab: Digital Equipment Corporation, 1984)

13. DG Luenberger, Y Ye, Constrained Minimization Coditions, in Linear and Nonlinear Programming, 3rd edn, Springer Press, New York, 321-354, (1973)

doi:10.1186/1687-1499-2012-236

Cite this article as: Liu et al:: Rate allocation based on spectrum pricing function in collaborative transmission over heterogeneous wireless access networks. EURASIP Journal on Wireless Communications and Networking 2012 2012:236.

\section{Submit your manuscript to a SpringerOpen ${ }^{\odot}$ journal and benefit from:}

- Convenient online submission

- Rigorous peer review

- Immediate publication on acceptance

- Open access: articles freely available online

- High visibility within the field

- Retaining the copyright to your article

Submit your next manuscript at $>$ springeropen.com 